Cathleen Grunert*

Katja Ludwig*

\title{
Erziehungswissenschaftliche Hauptfachstudiengänge in Deutschland. Zum Wandel von Studium und Disziplin im Zuge der Bolognareform
}

\begin{abstract}
Kurzusammenfassung
Der Beitrag befasst sich mit den europäischen Hochschulrefomprozessen und fokussiert dabei auf den sogenannten Bolognaprozess im Zuge dessen an den deutschen Universitäten eine flächendeckende Umwandlung der ehemaligen Diplom- und Magisterstudiengänge in Bachelor- und Masterstudiengänge stattgefunden hat. Auf der Basis einer Bestandsaufnahme der im Wintersemester 2014/2015 in erziehungswissenschaftlicher Verantwortung angebotenen Hauptfachstudiengänge steht dabei die Frage im Zentrum, wie sich die Studienlandschaft im Bereich der universitären Erziehungswissenschaft in Deutschland im Zuge der Bologna-Reform verändert hat. Daran schließt sich die Diskussion an, welche Konsequenzen die Einführung des Bachelorund Mastersystems für die institutionelle Organisation der Disziplin Erziehungswissenschaft in Form ihrer Studiengänge und damit auch für ihre Stellung im deutschen Hochschulsystem nach sich ziehen könnte.
\end{abstract}

Schlüsselwörter: Erziehungswissenschaft, Hochschule, Bologna-Prozess, Disziplin, Studiengänge.

\section{Educational Science in Germany: Changes in the Mode of Studying and in the Status of the Discipline as a Result of the Bologna Reform}

\begin{abstract}
This article deals with the European reorganization processes in higher education and in particular the so-called Bologna reform. In the case of German educational science, the Bologna Reform signifies a complete transition from the former study programs that end with a Diploma or Magister exam to the new system of Bachelor
\end{abstract}

* Fern-Universität in Hagen. 
and Master degrees. Based on a full survey of current major study programs offered in the area of educational science this article focuses on how the Bologna reform affects the landscape of study programs in educational science. That leads to the question of the consequences for the organization of Educational Science with regard to the academic courses offered and thus for the position of the discipline in the German university system.

Keywords: Educational Science, University, Bologna reform, Academic Disciplines, Study Programs.

\section{Die Heterogenität der Ausbildungssituation in der deutschen Erziehungswissenschaft}

„Es gibt viele Erziehungswissenschaftler in Deutschland, aber sie sind über alles verschiedener Meinung" (zit. nach Winkel 1989: 63) so konstatiert Hellmut Becker anlässlich der öffentlichen Vorstellung der Enzyklopädie Erziehungswissenschaft bereits 1983 die Lage der Disziplin in Westdeutschland. Das damit verbundene Bild einer heterogenen und unbestimmten Disziplin begleitete von Beginn an auch den Prozess der universitären Etablierung erziehungswissenschaftlicher Hauptfachstudiengänge (vgl. Grunert 1999; Grunert 2012).

Geht man davon aus, dass eine wissenschaftliche Disziplin nach Rudolf Stichweh (vgl. 1993: 241) als eine Forschungs- und Kommunikationsgemeinschaft von Wissenschaftlern gefasst werden kann, die durch gemeinsame Problemstellungen und Forschungsmethoden zusammengehalten wird, dann stellt sich zwangsläufig die Frage, was denn die Gemeinsamkeiten eigentlich sind, die diejenigen Studiengänge einigen, die sich im Kontext der Erziehungswissenschaft verorten. Und fügt man mit Stichweh (1994: 17) hinzu, dass, um von einer Disziplin sprechen zu können, Strukturen notwendig sind, die eine auf die Disziplin bezogene Karriere und fest eingerichtete Prozesse der Sozialisation ermöglichen, dann muss man zudem fragen, ob es diese festen Strukturen in der Erziehungswissenschaft überhaupt gibt und wie diese beschaffen sind.

Die Erziehungswissenschaft in Deutschland ist eine Disziplin, in der Identitätsdebatten auf Dauer gestellt zu sein scheinen, so dass auch die Frage nach dem disziplinären Kern oder dem einenden Band immer wieder neu gestellt wird (vgl. Krüger, Rauschenbach 1994; Osterloh 2002; Altefix 2009; Horn 2014). Drei Hypothesen erscheinen in aktueller Perspektive zentral für die damit verbundene starke Ausdifferenzierung und Diversifizierung der Erziehungswissenschaft als Disziplin und als Ausbildungsfach (Züchner, Rauschenbach 2016; Grunert 2012).

1. Ein erster Grund ist sicher der Hybridstatus der Erziehungswissenschaft, der sich aus ihrem unbestimmten Praxisbezug ergibt. Die Frage, was die Erziehungswissenschaft als Wissenschaftsdisziplin für die pädagogische Praxis überhaupt leisten kann, auf welche Praxisfelder sie sich beziehen soll und was die pädagogische Praxis überhaupt ist, auch darüber besteht keine 
Einigkeit (Krüger, Rauschenbach 2012; Horn 2014). Gleichzeitig ist die Erziehungswissenschaft immer wieder mit Anforderungen von außen konfrontiert, die das Modell einer reinen Beobachtungswissenschaft immer wieder in Frage stellen und sie in ihren Autonomieansprüchen herausfordern. Die Einführung von Bachelorstudiengängen, die in erster Linie berufsbefähigend sein sollen, erfordert zudem den Entwurf einer Praxis, der gleichzeitig die Gefahr in sich birgt, Studiengänge stark am aktuellen Markt orientiert zu entwerfen und Inhalte, deren Praxisrelevanz nicht unmittelbar sichtbar werden, zu verdrängen.

2. Ein zweiter Grund ist die starke Ausdifferenzierung der Erziehungswissenschaft in eine Reihe von Teildisziplinen (Krüger, Rauschenbach 2012). Dabei sind die Sozialpädagogik, die Erwachsenenbildung oder auch die Sonderbzw. Rehabilitationspädagogik klassische Teildisziplinen mit einer längeren Tradition, die sich mittlerweile auch auf eigene theoretische Bezugssysteme stützen können. Andere Teildisziplinen, wie vor allem die Pädagogik der frühen Kindheit konnten sich erst in den letzten Jahren breiter etablieren. Daneben existieren auch weniger ausgebaute und theoretisch wie methodisch geringer entwickelte Strömungen, die häufig konjunkturabhängig aufgrund aktueller Entwicklungen in der Praxis und weniger vor dem Hintergrund disziplininterner Prozesse zustande kommen (vgl. Altefix 2009: 47f.; Grunert 2012). Beide, Teildisziplinen und Strömungen, wie etwa Medienbildung oder Gesundheitsförderung, münden mittlerweile auch in eigene Studiengänge. Wenngleich diese Vielfalt einerseits grundsätzlich zu begrüßen ist - ist sie doch ein Anzeichen für eine zunehmende Professionalisierung pädagogischer Tätigkeitsfelder sowie einen Ausbau erziehungswissenschaftlicher Zuständigkeiten - stellt sich dennoch die Frage nach der disziplinären Ausrichtung solcher spezialisierter Studiengänge.

3. Drittens sind es sicher auch die herrschenden lokalen Wissenschaftskulturen, die auch vor der Bologna-Reform für differente Ausbildungsinhalte gesorgt haben und die die jeweilige inhaltliche Ausgestaltung der Studiengänge mitbestimmen. Differente Perspektiven auf relevante Theorien, Forschungsmethoden und Problemstellungen bilden zwar im heutigen Wissenschaftsverständnis das unhintergehbare Prinzip, bleiben aber, sofern sie in den Studiengängen nicht auf einen grundlegenden Kern aufbauen, letztlich ohne disziplinären Ort.

\section{Ein Kerncurriculum zur Sicherung disziplinärer Mindeststandards?}

Die Bologna-Reform und die damit verbundene Umstellung aller ehemaligen Diplomstudiengänge auf das Bachelor-/Master-System entfachte in der deutschen Erziehungswissenschaft erneut eine Diskussion um ein verbindendes Kerncurriculum für erziehungswissenschaftliche Studiengänge. Damit verband sich nicht nur 
die Hoffnung, die heterogene Ausbildungssituation zu bearbeiten, sondern auch die Identität und das Selbstverständnis der Erziehungswissenschaft als eigenständige universitäre Disziplin zu stärken sowie ihren Stellenwert gegenüber der Lehrerbildung zu betonen (Austermann et al. 2004: 38; Nieke 2007: 30).

Jedoch war in der deutschen Erziehungswissenschaft bereits die Rahmenordnung für den erziehungswissenschaftlichen Diplomstudiengang umstritten, so dass auch das erstmals 2004 von der DGfE veröffentliche Kerncurriculum Erziehungswissenschaft (DGfE 2004, 2010) nicht kritiklos aufgenommen und als Bezugsgröße für die Ausgestaltung von Studienprogrammen in Frage gestellt wurde. Im Jahre 2008 erfuhr das KCE eine nochmalige Überarbeitung und ist als inhaltliche und strukturelle Empfehlung der Fachgesellschaft DGfE zu betrachten, das kein Gesamtcurriculum definiert, sondern lediglich Mindeststandards formuliert, die einen erziehungswissenschaftlichen Studiengang ausmachen. Auf konkrete Vorgaben in Bezug auf theoretische und methodische Ansätze wird dabei bewusst verzichtet.

Die Kritik, ein Kerncurriculum würde die methodische und theoretische Pluralität gefährden und die Erziehungswissenschaft zu einer statischen, nicht wandelbaren Disziplin machen, erscheint damit unbegründet. Vielmehr macht die Suche nach dem Verbindenden in der Pluralität sie überhaupt erst zu einer Disziplin, liegt doch nur darin die Chance, gemeinsame Problemstellungen zu benennen und eine Kommunikationsgemeinschaft im Sinne Stichwehs zu identifizieren, die ihre Probleme und Themen nicht nur von außen zugewiesen bekommt (vgl. Hoffmann 2011: 228), sondern eigendynamisch entwickelt. Die Arbeit an einem gemeinsamen Kerncurriculum lässt sich damit auch als „boundary-work“ (Gieryn 1983; Beer, König, 2009) fassen, als Arbeit an eben dem gemeinsamen Kern, der einer Wissenschaftsdisziplin Kontur verleiht. Gleichzeitig lässt sich nur so vermeiden, dass „die Schnittmenge des gemeinsamen Wissens von Absolventen erziehungswissenschaftlicher Studiengänge quasi gegen Null tendiert" (bereits Vogel 1994: 380).

\section{Methodische Anmerkungen}

Die nachfolgenden Darstellungen basieren auf Erhebungen im Rahmen des von der DFG geförderten Projekts „Erziehungswissenschaft im Bologna-Prozess“ innerhalb dessen u.a. eine Bestandsaufnahme aller in erziehungswissenschaftlicher Verantwortung getragener Hauptfachstudiengänge ${ }^{1}$ vorgenommen wurde, in die zum Wintersemester 2014/2015 eine Immatrikulation in das erste Fachsemester möglich war (vgl. auch Grunert, Ludwig et al. 2016; Grunert, Ludwig 2016). Hierfür wurden auf der Basis vorliegender Bestandsaufnahmen zu Hochschulstandorten

\footnotetext{
${ }^{1}$ Als Hauptfachstudiengang wurde ein Studiengang gezählt, wenn es möglich ist, die Abschlussarbeit in Erziehungswissenschaft zu verfassen. Studiengänge, die auf ein Lehramt zielen, weiterbildend oder berufsbegleitend sind, wurden nicht berücksichtigt.
} 
mit erziehungswissenschaftlichen Studiengängen (vgl. etwa Krüger et al. 2003; Stisser, Horn 2012) sowie eigenen Vorerhebungen (Grunert 2012) ergänzende Internetrecherchen durchgeführt. Neben den Angaben im Hochschulkompass ${ }^{2}$ wurden dabei jeweils die Internetseiten der erziehungswissenschaftlichen Institute bzw. Fakultäten an Universitäten und Pädagogischen Hochschulen in Deutschland gesichtet und die Prüfungs- und Studienordnungen sowie Modulhandbücher und Studienverlaufspläne erhoben.

Ausgewertet wurden einerseits Strukturdaten zu den Studiengängen, wie die Art des Studiengangs (Bachelor oder Master), die Anzahl der Fächer (Ein-Fach oder Zwei-Fach-Studiengänge ${ }^{3}$ ), die Studienfachbezeichnung und Studienumfänge (in Leistungspunkten). Andererseits erfolgte in Anlehnung an das Kerncurriculum der DGfE und auf der Basis einer quantitativen Inhaltsanalyse eine Erhebung der inhaltlichen Anteile der Studienprogramme (in SWS und LP4). Dieser Beitrag richtet nun seine Aufmerksamkeit auf den Ausschnitt der Strukturdaten sowie den Vergleich der vorhandenen Anteile an Grundlagen der Erziehungswissenschaft und an Forschungsmethoden in den Studienprogrammen mit den Empfehlungen des Kerncurriculums.

\section{Erziehungswissenschaftliche Bachelor- und Masterstudiengänge in Deutschland}

Zum Wintersemester 2014/2015 finden sich in Deutschland an insgesamt 63 Standorten erziehungswissenschaftliche Hauptfachstudiengänge. Waren 2001, und damit vor der Bologna-Reform noch an 66 Standorten erziehungswissenschaftliche Hauptfachstudiengänge vorhanden (Krüger et al. 2003; Krüger, Rauschenbach 2004), so lässt sich damit ein Abbau von 3 erziehungswissenschaftlichen Standorten verzeichnen. Zudem finden sich an nur 54 Standorten sowohl Bachelor- als auch Masterstudiengänge, die im Sinne einer erziehungswissenschaftlichen Langzeitausbildung, wie sie in den Diplomstudiengängen gegeben war, überhaupt erst eine Vergleichbarkeit mit der Situation vor der Studienreform ermöglichen. Nach wie vor lässt sich damit ein Abbau erziehungswissenschaftlicher Standorte von immerhin knapp 20\% feststellen, so dass sich allein die Umstellung auf die neuen Studiengänge als Schrumpfungsfaktor für die Disziplin erweist.

Betrachtet man aber die absolute Anzahl der neuen Studiengänge im erziehungswissenschaftlichen Hauptfach, so bekommt man den Eindruck einer massiven Expansion. Deutschlandweit finden sich insgesamt 190 Bachelor- und Master-

\footnotetext{
${ }^{2}$ www.hochschulkompass.de

${ }^{3}$ Studiengänge mit Nebenfächern von mind. 30 LP (i.d.R. entspricht dies einem Semester) wurden als Zwei-Fach-Studiengänge eingeordnet.

${ }^{4}$ SWS $=$ Semesterwochenstunde; LP = Leistungspunkte.
} 
Studiengänge. Davon sind 86 Bachelor- und 104 Masterstudiengänge. Diese hohen Zahlen kommen zum einen nur auf Basis der Zweiteilung der ehemaligen Langzeitausbildung zustande und sind zweitens durch häufig vorhandene parallele Angebote an Ein- und Zwei-Fach-Studiengängen an einem Standort bedingt (vgl. Abb. 1). So finden sich im Bachelor nahezu ähnlich häufig Ein-Fach- und Zwei-FachStudiengänge (52\% bzw. 48\%) während sich im Master das Ein-Fach-Modell durchgesetzt hat (92\%).

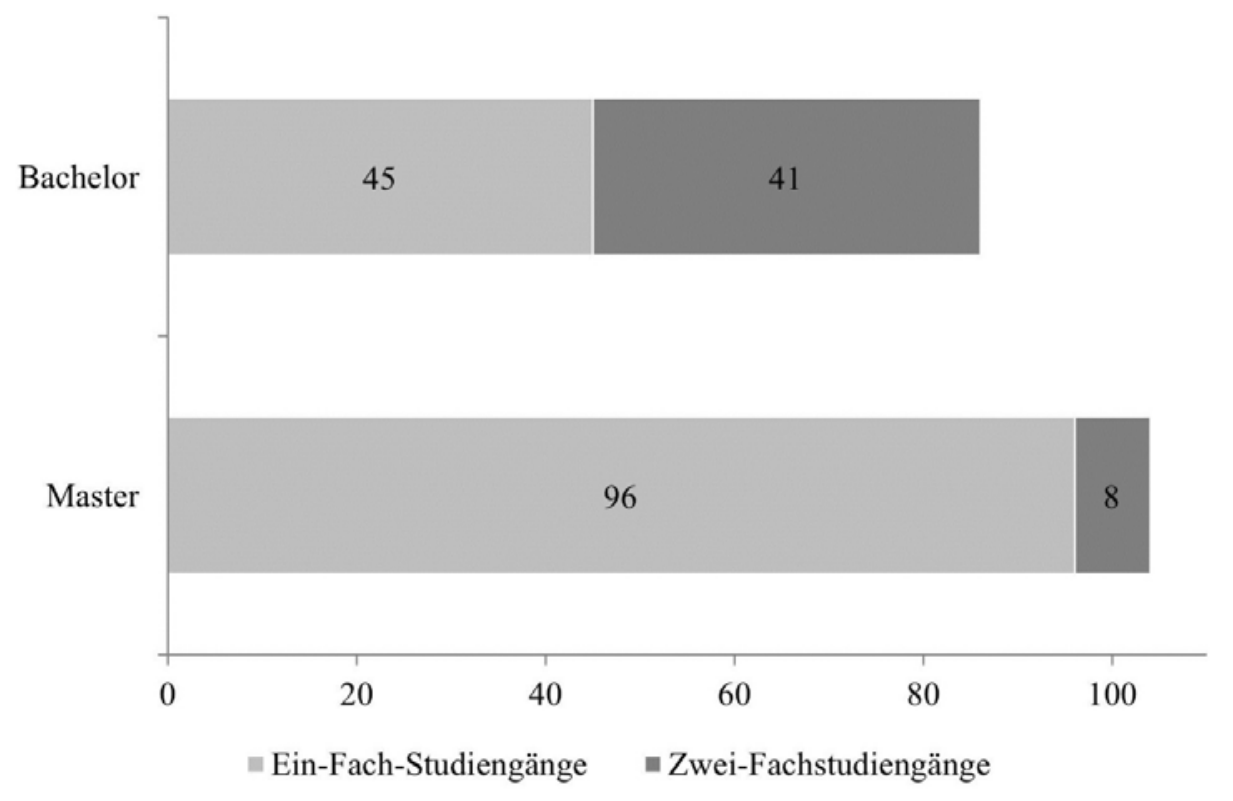

Abbildung 1. Ein- und Zwei-Fach-Studiengänge im Bachelor und Master ( $\mathrm{n}=190$, absolute Häufigkeiten)

Auf der Bachelorebene finden sich 25 Standorte, die ausschließlich Ein-FachStudiengänge anbieten. An 23 Standorten ist die Erziehungswissenschaft nur innerhalb von Zwei-Fach-Bachelor-Studiengängen vertreten, was gleichzeitig auf einen reduzierten Anteil erziehungswissenschaftlicher Inhalte verweist. 20 Standorte bieten jedoch auch ein Mehrfachangebot von mehr als einem BachelorStudiengang mit erziehungswissenschaftlichen Anteilen an, wenngleich hiervon lediglich 8 Standorte sowohl Ein-Fach- als auch Zwei-Fach-Varianten und nur 13 Standorte mehr als einen BA-Studiengang mit einem Umfang von $120^{5}$ oder mehr Leistungspunkten vorhalten. Auf der Masterebene finden sich demgegenüber

\footnotetext{
${ }^{5}$ Aus dem Umfang erziehungswissenschaftlicher Anteile wurden Nebenfächer und Studium Generale herausgenommen und im Fall von Zwei-Fach-Studiengängen wurden auch außerhalb des Gesamtumfangs liegende Umfänge für die Abschlussarbeit und Pflichtpraktika eingerechnet.
} 
primär Ein-Fach-Studiengänge. Lediglich einen Zwei-Fach-Studiengang hält hier nur ein Standort vor. Demgegenüber existiert hier mit 29 auch eine ganze Reihe von Standorten, die mehr als einen Masterstudiengang anbieten. 18 von diesen verfügen mindestens über zwei Masterstudiengänge mit 120 LP erziehungswissenschaftlicher Anteile.

Im Durchschnitt gibt es pro Standort 3 Studiengänge, wobei die standortbezogene Anzahl von einem bis zu sieben Studiengängen reicht. Spitzenreiter sind hier die Universität zu Köln und die Pädagogische Hochschule Ludwigsburg. Während in Ludwigsburg der Studienfachbezeichnung nach drei verschiedene Bachelor- und vier verschiedene Masterstudiengänge angeboten werden, finden sich in Köln auch unterschiedliche Umfänge für ähnliche Studiengänge, und zwar je ein BAStudiengang Erziehungswissenschaft mit 180 und 90 LP, ein BA Frühförderung mit 180 LP, sowie je ein Master in Erziehungswissenschaft mit 68 und 82 LP, ein MA Interkulturelle Kommunikation und Bildung mit 120 LP und ein MA Rehabilitationswissenschaften mit 120 LP.

Die deutliche Mehrzahl der Standorte bietet sowohl einen Master als auch einen Bachelorstudiengang an. Nur an zwei Standorten werden ausschließlich Bachelor-Studiengänge vorgehalten. Einzelstandorte, an denen nur ein Masterstudiengang angeboten wird, finden sich mit sieben Standorten hingegen etwas häufiger. Diese strukturellen Unterschiede verweisen dabei auf zwei mögliche problematische Entwicklungen: so stellt sich bei fehlendem Masterstudiengang die Frage danach, wie an diesen Standorten fachbezogener wissenschaftlicher Nachwuchs rekrutiert und qualifiziert werden kann und bei fehlendem BachelorStudiengang ergibt sich für die Standorte das Problem, auf das Anwerben von Absolventen anderer Hochschulen angewiesen zu sein und sich auch auf unterschiedliche Ausgangsvoraussetzungen einstellen zu müssen.

Auffällig ist zudem, dass sich die Umfänge der einzelnen Hauptfachstudiengänge sehr stark unterscheiden. So liegen sie im BA-Bereich zwischen 70 und 210 und im MA-Bereich zwischen 68 und 120 LP. Insbesondere auf der Bachelorebene, auf der auch Ein- und Zwei-Fach-Studiengänge annähernd paritätisch verteilt sind, lassen sich dabei große Differenzen ausmachen. Insgesamt sind hier 14 verschiedene Umfänge festzustellen, die z.T. sehr „krumme Zahlen“ beinhalten, so dass sich bereits hier die Frage nach der Vergleichbarkeit und der mit der Studienreform vereinfachten Mobilität zwischen den einzelnen Studiengängen stellt. Eine Ausnahme bilden hier zudem zwei Bachelorstudiengänge, die als siebensemestrige Studiengänge angelegt sind und in 210 LP Gesamtumfang längere Praktikumszeiten integrieren.

Im Master, der vorwiegend als Ein-Fach-Studiengang angeboten wird, ist die Verteilung der Umfänge etwas eindeutiger, da hier die 120 LP als übergreifende Richtgröße dienen. Dennoch finden sich unterhalb dieser Marke immerhin fast ein Fünftel der Masterstudiengänge, wobei sich hier auch vier Studiengänge im Umfang 
von 90 LP finden, die als Ein-Fach-Studiengänge ausgelegt sind und ein Bachelorstudium im Umfang von $210 \mathrm{LP}$ voraussetzen.

Bereits die Darstellung der strukturellen Charakteristika der erziehungswissenschaftlichen Bachelor- und Masterstudiengänge in Deutschland macht deutlich, dass von einer sehr heterogenen Entwicklung auszugehen ist, die sich noch stärker in den Bachelor- als in den Masterstudiengängen zeigt und mit der im Vergleich zur Situation vor der Bologna-Reform auch ein leichter Abbau von Standorten mit erziehungswissenschaftlicher Hauptfachausbildung einhergegangen ist.

\section{Heterogenität von Fachbezeichnungen erziehungswissenschaft- licher Studiengänge}

Der Blick auf die Strukturdaten sagt jedoch noch nichts über die inhaltliche Ausrichtung der jeweiligen Studienprogramme aus. Eine erste Annäherung an inhaltliche Fragen ermöglicht der Blick auf die unterschiedlichen Fachbezeichnungen der erziehungswissenschaftlichen Studiengänge in Deutschland. Fachbezeichnungen dienen nicht nur der Orientierung für Studierende, sondern sind immer auch Symbole disziplinärer Grenzziehungen, die jedoch zunehmend auch in außerdisziplinäre Rahmenbedingungen, hochschulpolitische Entscheidungen und Wettbewerbslogiken eingebettet sind (Grunert, Ludwig 2016). Die Fachbezeichnungen der deutschen BA- und MA-Studiengänge in der Erziehungswissenschaft verweisen dabei auf sehr differente symbolische Grenzziehungen und verstärken das Bild einer heterogenen und stark ausdifferenzierten Disziplin einmal mehr.

So lassen sich bei den 86 Bachelor-Studiengängen insgesamt 35 verschiedene Fachbezeichnungen ausmachen. Im Masterbereich ist dies noch differenzierter, so dass sich hier bei 104 Studiengängen ganze 70 verschiedene Fachbezeichnungen finden. Neben der strukturellen Heterogenität deutet diese Vielfalt damit auch in inhaltlicher Perspektive auf eine deutlich plurale Studiengangslandschaft im Feld der Erziehungswissenschaft hin.

Sortieren lassen sich die Studiengänge ihren Fachbezeichnungen nach in Studiengänge mit einem eher generalisierten Label und Studiengänge mit einem eher spezialisierten Label. Als generalisierte Studiengänge wurden diejenigen gefasst, die die Fachbezeichnung Erziehungswissenschaft, Pädagogik, Bildungswissenschaft oder Kombinationen aus diesen Begriffen verwenden, ohne damit vorzugeben, dass darüber auf die tatsächlichen Studieninhalte geschlossen werden könnte. Im Bachelorbereich können diesem Kriterium insgesamt 63\% (54 von 86) und im Masterbereich 37\% (38 von 104) der Studiengänge zugeordnet werden.

Bereits diese Gruppe der generalisierten Studiengänge verweist auf eine sehr heterogene Verwendung von Fachbezeichnungen sowie eine häufig vorhandene parallele Verwendung unterschiedlicher Disziplinbegriffe. Zwar ist der Begriff der „Erziehungswissenschaft“ der dominanteste, wird aber auch mit den Begriffen 
Bildungswissenschaft oder Pädagogik parallel verendet (vgl. Abb. 2). Am zweithäufigsten findet sich die Fachbezeichnung Pädagogik, aber auch der Begriff der Bildungswissenschaft wird vor allem auf der Masterebene verstärkt verwendet. Zudem gibt es eine Reihe an Kombinationen der generalisierten Fachbezeichnungen Erziehungs- und Bildungswissenschaft sowie Pädagogik mit thematischen Schwerpunkten, was zumindest der Studienfachbezeichnung nach auf eine stärkere Spezialisierung verweist.

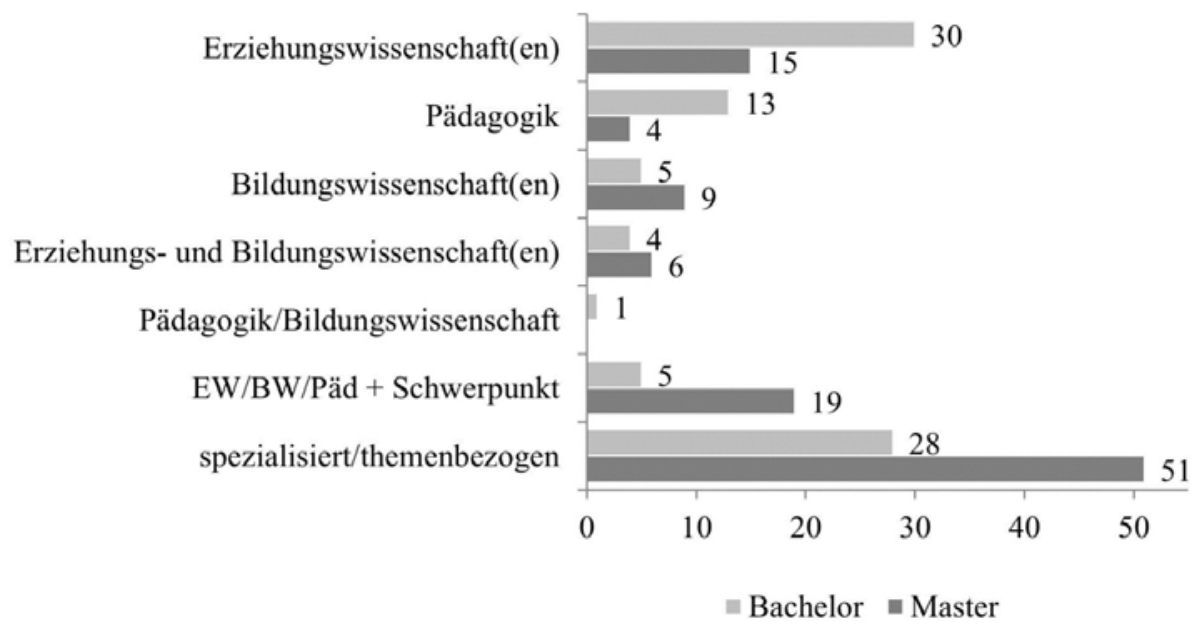

Abbildung 2. Studienfachbezeichnungen ( $\mathrm{n}_{\mathrm{BA}}=86 ; \mathrm{n}_{\mathrm{MA}}=104$; absolute Häufigkeiten)

Der Begriff der Erziehung erscheint damit als Mangelbegriff, der der Ergänzung bedarf und als alleinige Zuschreibung von Studiengängen nicht mehr ausreichend zu sein scheint. Ursachen dafür liegen vor allem in der zunehmenden Ausdifferenzierung erziehungswissenschaftlicher Gegenstände und pädagogischer Tätigkeitsfelder, die sich in den Studiengängen wiederfinden und die mit Begriff der Erziehung nicht mehr angemessen zu fassen sind (vgl. auch Liebau 2002: 293ff.). Ergänzungen zu generalisierten Fachbezeichnungen durch Schwerpunktnennungen finden sich bei insgesamt 24 Studiengängen. Teilweise sind diese eher allgemein gehalten, wie z.B. „Erziehungswissenschaft: Heterogenität in Erziehung und Bildung" und können damit der Fachbezeichnung nach in die Gruppe der generalisierten Studiengänge eingeordnet werden. Mehrheitlich verweisen diese Studiengänge jedoch auf spezifische Themenbereiche, wie z.B. Organisationsentwicklung oder auf teildisziplinäre Schwerpunkte, wie z.B. Sozialpädagogik oder Pädagogik der Kindheit, die der Gruppe spezialisierter Studiengänge zugeordnet wurden.

Gerade über die parallele Verwendung unterschiedlicher Disziplinbegriffe erscheinen die symbolischen Grenzziehungen jedoch sehr diffus, vor allem dann, wenn unterschiedliche Fachbezeichnungen nicht mit ähnlichen inhaltlichen Ausrichtungen einhergehen. 


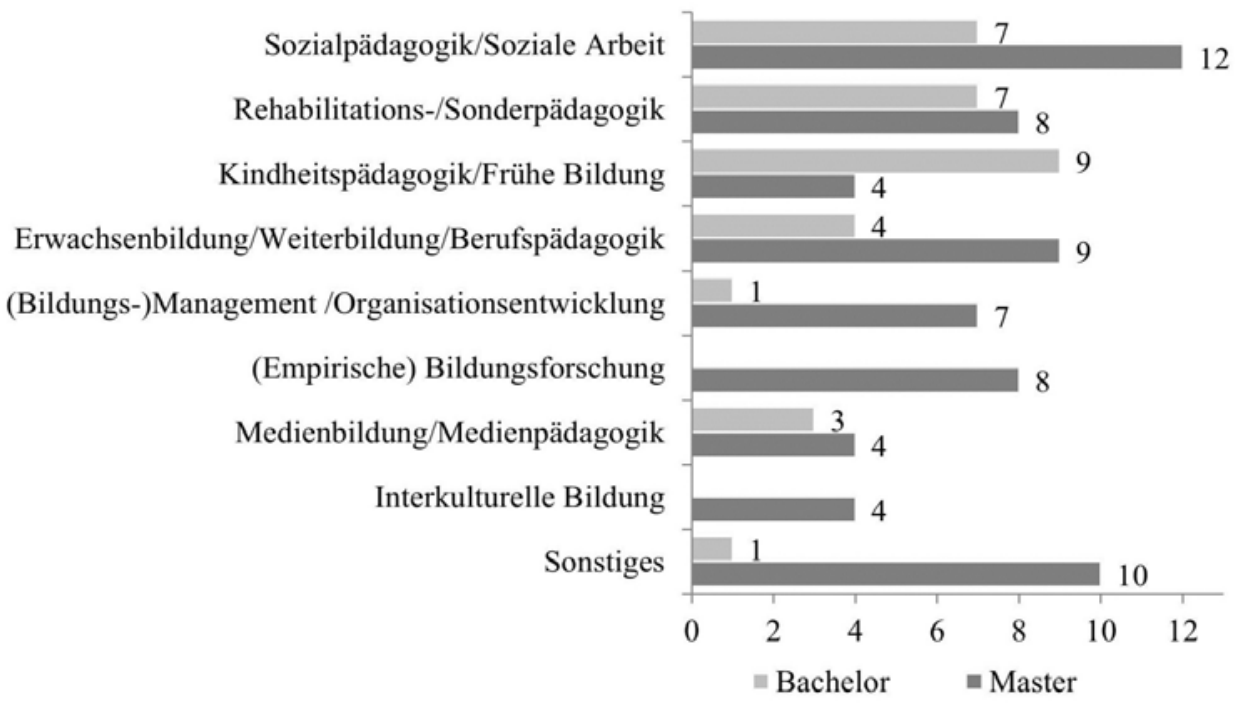

Abbildung 3. Spezialisierte Studienfachbezeichnungen $\left(\mathrm{n}_{\mathrm{BA}}=32 ; \mathrm{n}_{\mathrm{MA}}=66\right.$; absolute Häufigkeiten)

Bei den spezialisierten Studiengängen (vgl. Abb. 3) wurde eine Gruppierung nach ähnlichen Profilen vorgenommen, wobei damit auch die hier ausgeprägte Heterogenität von Studienfachbezeichnungen selbst bei ähnlichen inhaltlichen Spezialisierungen verdeckt wird. Deutlich wird dennoch, dass sich aus einigen der bisherigen Studienrichtungen, die im erziehungswissenschaftlichen Diplomstudiengang die berufsfeldbezogenen Anteile im Gesamtstudienkonzept Erziehungswissenschaft ausgemacht hatten, eigene Studiengänge entwickelt haben. Dies gilt insbesondere für die Rehabilitations- und Sonderpädagogik sowie die Sozialpädagogik sowohl auf Bachelor- als auch auf Masterebene, während die Erwachsenenbildung mit eigenen Studiengängen vor allem auf der Masterebene vertreten ist.

Zudem wird deutlich, dass der Bereich der Kindheitspädagogik den größten Ausbau erfahren hat. Die Debatten um die Anhebung der beruflichen Qualifikation in den entsprechenden Handlungsfeldern in Verbindung mit der Bologna-Reform scheinen hier als Katalysatoren einer Umwandlung der früheren Studienrichtungen im Diplomstudiengang in eigenständige akademische Studiengänge fungiert zu haben.

Im Master lassen sich darüber hinaus auch Studiengänge finden, die einen starken Forschungsbezug aufweisen sowie eine Reihe von Studiengängen, die nicht den etablierten Teildisziplinen zuzuordnen sind, wie etwa der Studiengang „Abenteuer- und Erlebnispädagogik“ in Marburg oder der Studiengang „Bildung in Europa" an der Universität Flensburg.

Die erziehungswissenschaftlichen Bachelor-Studiengänge sind insgesamt zu gut einem Drittel als spezialisierte Studiengänge einzuordnen, während auf Mas- 
terebene der Fachbezeichnung nach immerhin zu 63\% spezialisierte Studiengänge auszumachen sind.

Neben einer Reihe von unterschiedlichen Fachbezeichnungen findet sich innerhalb der Studiengänge auch eine Vielzahl an möglichen Schwerpunktsetzungen in wiederum stark differierenden Umfängen. Während in 35 der 53 generalisierten Bachelorstudiengänge eine Schwerpunktsetzung erfolgt, trifft dies im Masterbereich auf 34 der 37 Studiengänge $\mathrm{zu}^{6}$. Dabei finden sich allein in den 35 Bachelorstudiengängen 142 unterschiedliche Bezeichnungen für wählbare Schwerpunkte, die sich nur noch in geringen Anteilen an der traditionellen teildisziplinären Fachsystematik orientieren. Zudem haben die Schwerpunkte an den einzelnen Standorten einen unterschiedlichen Stellenwert, so dass in einigen Fällen mehrere Schwerpunkte gewählt werden müssen oder unterschiedlich viele Schwerpunkte zur Auswahl stehen oder in anderen Fällen die Schwerpunktsetzung nur über die Ausrichtung von Praktikum und Bachelorarbeit erfolgt. Der Versuch einer Gruppierung zeigt deutlich eine starke Position der Erwachsenenbildung/Weiterbildung (26), die ihre Studienschwerpunkte mit relativ einheitlichen Fachbezeichnungen markiert. Daneben finden sich, wenn auch in geringeren Anteilen, die klassischen Studienrichtungen Sozialpädagogik (20) und Rehabilitationspädagogik (12) (vgl. auch Grunert, Ludwig et al. 2016). Demgegenüber verweisen diejenigen Schwerpunkte, die außerhalb dieser traditionellen teildisziplinären Kennzeichnung liegen auf eine starke Ausdifferenzierung in eine Reihe von Einzelschwerpunkten und zeichnen so eine Entwicklung nach, die auch für die Studiengänge generell ausgemacht werden konnte (Grunert 2012).

\section{Zur Relevanz des Kerncurriculums für erziehungswissenschaft- liche Studiengänge}

Wie bereits angedeutet, wurden die Studiengänge im Forschungsprojekt auch daraufhin befragt, inwieweit sie sich in der Ausgestaltung ihrer Studienpläne an dem von der DGfE vorgeschlagenen Kerncurriculum orientieren (vgl. Grunert, Ludwig 2016; Grunert, Ludwig et al. 2016). Im Bachelorbereich gliedert sich das KCE in 3 Studieneinheiten mit je zwei bis vier Teilbereichen und im Masterbereich umfasst das Kerncurriculum eine Studieneinheit mit drei Teilbereichen?.

Zur Darstellung der fachbezogenen Studieninhalte konzentrieren wir uns im Rahmen dieses Beitrags auf die grundlagenbezogenen Anteile (bezogen auf die

\footnotetext{
${ }^{6}$ Ab hier beziehen sich die Aussagen nur noch auf 184 Studiengänge, da ein Standort seinen Bachelorund Masterstudiengang zum Erhebungszeitpunkt noch nicht abschließend modularisiert hat und die Modulhandbücher nicht zur Verfügung standen.

${ }^{7}$ Hinzukommen curriculare Empfehlungen für unterschiedliche Studienrichtungen (BA: 36 LP; MA: 24 LP) sowie Empfehlungen für das Praktikum (BA: 30 LP; MA: 14 LP), die Abschlussarbeit (BA:10 LP; MA: 30 LP), Nebenfächer, Allgemeine Schlüsselqualifkationen und im Masterbereich für Lehrforschungsprojekte (vgl. DGfE 2010).
} 
Studieneinheit 1 im KCE für BA-Studiengänge: Grundbegriffe, Theorien, Geschichte und wissenschaftstheoretische Ansätze) sowie auf die Anteile für (quantitative und qualitative) Forschungsmethoden. Mit Blick auf strukturelle Empfehlungen für einen möglichen Umfang der Anteile bleibt das KCE jedoch sehr vage, da diese nur integriert neben anderen Studieneinheiten ${ }^{8}$ aus einem Pflicht- und einem Wahlbereich mit jeweils 26 LP ausgewiesen werden ${ }^{9}$. Geht man jedoch hypothetisch von einer symmetrischen Verteilung der Leistungspunkte im Pflichtbereich aus, dann sollten für den Bereich der Grundlagen wenigstens 9 LP in Bachelor-Studiengängen vorgesehen und für den Bereich der Forschungsmethoden mindestens 3 LP sowohl im BA als auch im MA integriert werden.

Bezogen auf die Anteile an den Grundlagen der Erziehungswissenschaft, wird deutlich, dass diese unterschiedlich stark in den Studienprogrammen vertreten sind (vgl. Tab. 1) und insgesamt fast ein Fünftel der Studiengänge (18\%) den Empfehlungen des KCE nicht folgt und keine dieser Inhalte vorsieht. In der Gruppe der generalisierten Bachelor-Studiengänge sind jedoch mit durchschnittlich 18,2 LP erwartbar die höchsten Anteile an Grundlagen integriert. Nur 4 dieser Studiengänge bleiben unter den Mindestanforderungen des Kerncurriculums Erziehungswissenschaft und immerhin zwei Drittel liegen deutlich darüber. Hier unterscheiden sich diejenigen Studiengänge mit der Fachbezeichnung „Bildungswissenschaft“ von denjenigen mit dem Label „Erziehungswissenschaft“ oder „Pädagogik“. Letztere integrieren mit durchschnittlich 18 bzw. 20 LP sehr hohe Anteile an Grundlagen, während erstere mit durchschnittlich 11 LP deutlich darunter liegen.

Tabelle 1. Grundlagenbezogene Anteile nach Leistungspunkten ( $\mathrm{n}=188$; in \%)

\begin{tabular}{|l|c|c|c|c|}
\hline $\begin{array}{c}\text { Leistungs- } \\
\text { punkte }\end{array}$ & $\begin{array}{c}\text { BA generalisiert } \\
(\mathrm{n}=53)\end{array}$ & $\begin{array}{c}\text { BA spezialisiert } \\
(\mathrm{n}=32)\end{array}$ & $\begin{array}{c}\text { MA generalisiert } \\
(\mathrm{n}=37)\end{array}$ & $\begin{array}{c}\text { MA spezialisiert } \\
(\mathrm{n}=66)\end{array}$ \\
\hline 0 LP & - & 25,0 & 8,1 & 51,5 \\
\hline 1-6 LP & 7,5 & 40,6 & 21,6 & 24,2 \\
\hline 7-12 LP & 24,5 & 18,8 & 40,5 & 10,6 \\
\hline 13-18 LP & 15,1 & 6,3 & 13,5 & 12,1 \\
\hline$\geq 19 \mathrm{LP}$ & 52,8 & 9,4 & 16,2 & 1,5 \\
\hline Durchschnitt & $18,2 \mathrm{LP}$ & $6,3 \mathrm{LP}$ & $10,5 \mathrm{LP}$ & $4,4 \mathrm{LP}$ \\
\hline
\end{tabular}

\footnotetext{
${ }^{8}$ Studieneinheit 2 = „Gesellschaftliche, politische und rechtliche Bedingungen von Bildung, Ausbildung und Erziehung in schulischen und nicht-schulischen Einrichtungen unter Einschluss internationaler Aspekte“ und Studieneinheit 3 = „Einführung in erziehungswissenschaftliche Studienrichtungen“ (vgl. DGfE 2010).

${ }^{9}$ Man könnte auch sagen, dass das KCE mit Blick auf die vorgeschlagenen Umfänge für ein disziplinbezogenes Grundlagenstudium deutlich so angelegt ist, dass den einzelnen Standorten genügend Spielraum für eine individuelle Ausgestaltung und Profilbildung ihrer eigenen Studiengänge bleibt. Allerdings lassen die Empfehlungen auch den Schluss zu, dass einzelne Teilbereiche lediglich mit einem Leistungspunkt abgedeckt werden können.
} 
Bachelor-Studiengänge mit spezialisierten Fachbezeichnungen bleiben im Vergleich dazu in Bezug auf die Vermittlung von Grundlagen der Erziehungswissenschaft mit durchschnittlich nur 6,3 LP weit unter den Mindestanforderungen des KCE. Acht Studiengänge verzichten dabei ganz auf eine erziehungswissenschaftliche Grundlagenausbildung, so dass in diesen Studiengängen die je spezifischen Bereiche und Praxisfelder im Zentrum der Ausbildung stehen und der Bezug zur Disziplin Erziehungswissenschaft diffus bleibt.

Auf der Ebene der Master-Studiengänge ist festzuhalten, dass - obgleich das KCE hier keine grundlagenbezogenen Inhalte empfiehlt - immerhin 92\% der generalisierten und 48\% der spezialisierten Studiengänge solche Inhalte vorsieht. Im Unterschied zu den Bachelorstudiengängen liegen die Leistungspunkte jedoch häufiger im unteren Bereich von 1 bis 6 LP, während sich größere Umfänge von 19 LP und mehr mit Ausnahme eines Studiengangs nur bei generalisierten Studiengängen finden. Bezogen auf die durchschnittlichen Anteile zeigen sich hier jedoch zwischen den verschiedenen generalisierten Fachbezeichnungen kaum noch deutliche Unterschiede.

Tabelle 2. Forschungsmethodenbezogene Anteile nach Leistungspunkten ( $\mathrm{n}=188$; in \%)

\begin{tabular}{|l|c|c|c|c|}
\hline $\begin{array}{c}\text { Leistungs- } \\
\text { punkte }\end{array}$ & $\begin{array}{c}\text { BA generalisiert } \\
(\mathrm{n}=53)\end{array}$ & $\begin{array}{c}\text { BA spezialisiert } \\
(\mathrm{n}=32)\end{array}$ & $\begin{array}{c}\text { MA generalisiert } \\
(\mathrm{n}=37)\end{array}$ & $\begin{array}{c}\text { MA spezialisiert } \\
(\mathrm{n}=66)\end{array}$ \\
\hline 0 LP & - & 15,6 & 13,9 & 31,8 \\
\hline 1-6 LP & 7,5 & 21,9 & 5,6 & 13,6 \\
\hline 7-12 LP & 47,2 & 46,9 & 30,6 & 31,8 \\
\hline 13-18 LP & 20,8 & 12,5 & 22,2 & 10,6 \\
\hline$\geq 19 \mathrm{LP}$ & 24,5 & 3,1 & 27,8 & 12,1 \\
\hline Durchschnitt & $13,8 \mathrm{LP}$ & $7,9 \mathrm{LP}$ & $13,3 \mathrm{LP}$ & $8,5 \mathrm{LP}$ \\
\hline
\end{tabular}

Im Hinblick auf die Forschungsmethoden ergibt sich ein ähnlich heterogenes Bild (vgl. Tab. 2). Hier erweisen sich die generalisierten Studiengänge stärker forschungsorientiert als die spezialisierten, bei denen fünf BA- und 21 MAStudiengänge ganz auf eine Ausbildung in Forschungsmethoden verzichten. Mit Blick auf das Kerncurriculum muss jedoch gesagt werden, dass wenn Forschungsmethoden in die Studiengänge integriert sind, diese auch mindestens mit 3 LP berücksichtigt werden. Dennoch kann, angesichts der stark schwankenden Anteile im Bachelorbereich, die Vorbereitung der Studierenden auf spätere Forschungstätigkeiten sowie auf eine wissenschaftliche Weiterqualifikation etwa in Masterstudiengängen durchaus mit Skepsis betrachtet werden. Zudem lässt sich danach fragen, inwiefern die Masterstudiengänge selbst auf Forschungstätigkeiten vorbereiten, schwanken hier nicht nur die Umfänge, sondern konzentrieren sich insbesondere bei den spezialisierten Studiengängen diejenigen, die ganz auf Forschungsmetho- 
den verzichten und stärker handlungsfeldbezogen ausgerichtet sind. Insgesamt sieht etwa die Hälfte der Studiengänge sowohl eine quantitative als auch qualitative Methodenausbildung vor und folgt damit auch in inhaltlicher Hinsicht den Empfehlungen der DGfE. Allerdings überwiegen Studienprogramme mit einer stärkeren Ausrichtung auf quantitative Methoden (BA: 42\%; MA: 22\%), während sich eine qualitative Fokussierung lediglich in vier Masterstudiengängen findet.

\section{Fazit}

Insgesamt geht das Aufeinandertreffen hochschulpolitischer Reorganisationsprozesse mit einer identitätssuchenden Disziplin mit einschneidenden Veränderungen für die deutsche Erziehungswissenschaft einher, die hier vor allem anhand der institutionellen Organisation der Erziehungswissenschaft in Form ihrer Hauptfachstudiengänge deutlich gemacht werden konnten.

Dieser Prozess führt nicht nur zu einem Abbau von Standorten, die erziehungswissenschaftliche Hauptfachstudiengänge anbieten sondern auch zu einer Heterogenisierung der strukturellen Merkmale der Studiengänge sowie zu einer deutlichen Ausdifferenzierung von Studieninhalten, Studienprofilen und vor allem auch Fachbezeichnungen für Studiengänge (vgl. auch Grunert 2012; Stisser, Horn 2012, Grunert, Ludwig et al. 2016). Mit zunehmender Spezialisierung und Differenzierung stellt sich dann auch die Frage nach den disziplinären Bezügen der Studiengänge, da in der Gesamtschau ein Kerncurriculum und verbindende Fachbezeichnungen kaum noch auszumachen sind.

Welche Konsequenzen jedoch der aktuell eingeschlagene Weg einer starken Heterogenisierung und Spezialisierung tatsächlich in einer langfristigen Perspektive für die Disziplin haben wird, muss dringend weiter beobachtet und diskutiert werden, insbesondere unter der Maßgabe, dass die ausbildungsbezogenen Bestandteile der Disziplin nun nicht einmal mehr das Label erziehungswissenschaftlich gemeinsam haben. Vor dem Hintergrund der - trotz erfolgreicher Institutionalisierung (vgl. Züchner, Rauschenbach 2016) - nicht immer sicheren Stellung der erziehungswissenschaftlichen Hauptfachstudiengänge und ihrer oft vorhandenen Konkurrenz zur Lehrerbildung an den deutschen Hochschulen, kann dieser Verzicht auf eine starke symbolische Grenzziehung über einheitliche Fachbezeichnungen auf Dauer auch eine Schwächung der Stellung der Disziplin im Hochschulsystem bedingen, da die Erziehungswissenschaft dadurch immer weniger sichtbar wird. Gleichzeitig muss beobachtet werden, inwiefern nicht auch die Einrichtung unikater Einzelstudiengänge mit dem Ziel des Alleinstellungsmerkmals problematisch werden kann, insbesondere da sie als „Exoten“ hochschulpolitischen Sparmaßnahmen schneller zum Opfer fallen könnten (auch Grunert 2012).

Zudem ist mit der Bologna-Reform und der Etablierung eigener Studiengänge auch ein Erstarken der klassischen Teildisziplinen verbunden, in dessen Zuge auch 
immer wieder Diskussionen um eine disziplinäre Eigenständigkeit laut werden (vgl. z.B. Scherr 2010). Aber ebenso wie im Gesamtprozess der Transformation erziehungswissenschaftlicher Hauptfachstudiengänge scheint auch die teildisziplininterne Entwicklung nicht auf einem koordinierten Verfahren zu beruhen, zu heterogen sind auch innerhalb dieser Studiengänge die strukturellen und inhaltlichen Ausrichtungen wie auch die symbolischen Grenzziehungen über unterschiedliche Fachbezeichnungen.

Darüber hinaus stellt sich die Frage nach der zukünftigen Reproduktion des wissenschaftlichen Nachwuchses in der deutschen Erziehungswissenschaft vor allem angesichts der starken Konzentration auf die berufsfeldbezogenen Anteile sowie der Verzicht auf eine flächendeckende Ausbildung in Forschungsmethoden v.a. in den spezialisierten Studiengängen. Gleichzeitig vergrößert sich damit auch die Gefahr der Verlagerung von Studiengängen an Fachhochschulen (vgl. auch Krüger 2010: 334).

Der Ausdifferenzierungsprozess kann aber auch die Chance für eine Neuordnung der Disziplin sowie einen weiteren Ausbau akademisch gebundener erziehungswissenschaftlicher Zuständigkeiten in sich bergen. Dafür müsste jedoch die Ausweitung erziehungswissenschaftlicher Zuständigkeiten in der Ausbildung an eine Diskussion über ein gemeinsames Selbstverständnis auch im Sinne eines Kerncurriculums gekoppelt werden. Dass die Studienreform in der Erziehungswissenschaft dazu bislang kaum beigetragen hat und in der Ausgestaltung der Studiengänge kaum ein Minimalkonsens zu finden ist, hat der Beitrag gezeigt. So erscheint die Studienreform als Katalysator für eine exzessive Ausdifferenzierung erziehungswissenschaftlicher Studiengänge, die die disziplinäre Kommunikation aktuell erstaunlich unberührt lässt. Angesichts der skizzierten disziplinschwächenden Entwicklung von innen sowie der mehrfach bestehenden Drucksituation durch äußere Faktoren, wäre eine gemeinsame Verständigung über das Gemeinsame in der Vielfalt jedoch dringend geboten (vgl. auch Grunert 2012).

\section{Literaturverzeichnis}

Altefix T. (2009) Das Pädagogische jenseits von Erfahrung und Denken: ein erkenntnisethischer Begründungsversuch im Sinne Franz Fischers, Würzburg, Königshausen \& Neumann.

Austermann S., Freitag J., Vogel P., Wigger L. (2004) Kerncurriculum Erziehungswissenschaft - Konzepte und Erfahrungen, „Erziehungswissenschaft“, 15 (28): 37-48.

Beer B., Koenig M. (2009) Grenzziehungen im System wissenschaftlicher Disziplinen der Fall der „Kulturwissenschaft(en)“, „Sociologia Internationalis“, 47 (1): 3-38.

DGfE = Deutsche Gesellschaft für Erziehungswissenschaft (2004) Kerncurriculum für das Hauptfachstudium Erziehungswissenschaft, Online: http://www.dgfe.de/fileadmin/ OrdnerRedakteure/Stellungnahmen/2004_01_KC_HF_EW.pdf [zuletzt: 20.07.2016]. 
DGfE (2010) Kerncurriculum Erziehungswissenschaft. Empfehlungen der Deutschen Gesellschaft für Erziehungswissenschaft, 2. erw. Aufl., Opladen, Barbara Budrich.

Gieryn T. F. (1983) Boundary-Work and the Demarcation of Science from Non-Science: Strains and Interests in Professional Ideologies of Scientists, "American Sociological Review", 48 (6): 781-795.

Grunert C. (1999) Vom Pionier zum Diplom-Pädagogen. Lebensgeschichten und Berufsperspektiven von ostdeutschen Studierenden im Diplom-Studiengang Erziehungswissenschaft, Opladen: Leske+Budrich.

Grunert C. (2012) Erziehungswissenschaft im Spiegel ihrer Studiengänge. Die Studienreform und ihre Folgen für die Disziplin, "Zeitschrift für Erziehungswissenschaft", 15(2): 573-596.

Grunert C., Ludwig K. (2016) Disziplinen im Wandel? - Erziehungswissenschaft und Soziologie im Bolognaprozess, „Zeitschrift für Pädagogik“, 6/2016: 886-907.

Grunert C., Ludwig K., Radhoff M., Ruberg C. (2016) Studiengänge und Standorte, in: Datenreport Erziehungswissenschaft 2016, in: H.-C. Koller, H. Faulstich-Wieland, H. Weishaupt, I. Züchner (ed.), Opladen, Barbara Budrich: 19-69.

Hoffmann D. (2011) Bildungspolitik und Wissenschaftspolitik, in: Ders. Kritische Wissenschaftsforschung: Untersuchungen zur gesellschaftlichen Bedingtheit und ideologischen Befangenheit pädagogischer Theorie und Praxis, in: D. Hoffmann (ed.), Hamburg, Kovac: 207-231.

Horn K.-P. (2014) Pädagogik/Erziehungswissenschaft der Gegenwart. Zur Entwicklung der deutschen Erziehungswissenschaft im Spiegel ihrer disziplinären Selbstreflexion (1910 - 2010), in: Das Selbstverständnis der Erziehungswissenschaft. Geschichte und Gegenwart, „Zeitschrift für Pädagogik“, 60. Beiheft, R. Fatke, J. Oelkers (ed.), Weinheim, Basel, Beltz, Juventa: 14-32.

Krüger H.-H. (1994) Allgemeine Pädagogik auf dem Rückzug? Notizen zur disziplinären Neuvermessung der Erziehungswissenschaft, in: Erziehungswissenschaft. Die Disziplin am Beginn einer neuen Epoche, in: H.-H. Krüger, T. Rauschenbach (ed.), Weinheim, Juventa: $115-130$.

Krüger H.-H. (2010) Erziehungswissenschaft und Sozialpädagogik, in: Grundriss Soziale Arbeit, in: W. Thole (ed.), 3. überarb. u. erw. Aufl., Wiesbaden: VS Verlag: 325-336.

Krüger H.-H., Rauschenbach T. (1994) Erziehungswissenschaft. Die Disziplin am Beginn einer neuen Epoche, Weinheim, Juventa.

Krüger H.-H., Rauschenbach T. (2004) Pädagogen in Studium und Beruf. Empirische Bilanzen und Zukunftsperspektiven, Wiesbaden: VS Verlag. 
Krüger H.-H., Rauschenbach T. (2012) Einführung in die Arbeitsfelder des Bildungsund Sozialwesens, 5. Aufl., Opladen, Toronto, Barbara Budrich.

Krüger H.-H., Rauschenbach T., Grunert C. et al. (2003) Diplom-Pädagogen in Deutschland. Survey 2001, Weinheim, Juventa.

Liebau E. (2002) Bildungswissenschaft. Zur Weiterentwicklung der Disziplin, „Vierteljahrsschrift für wissenschaftliche Pädagogik“, 78: 293-299.

Nieke W. (2007) Ausdifferenzierung und Kapazitätsprobleme. Hauptfachstudiengänge der Erziehungswissenschaft, „Erziehungswissenschaft“, 18 (35): 25-37.

Osterloh J. (2002) Identität der Erziehungswissenschaft und pädagogische Verantwortung. Ein Beitrag zur Strukturdiskussion gegenwärtiger Erziehungswissenschaft in Auseinandersetzung mit Wilhelm Flitner, Bad Heilbrunn, Klinkhardt.

Scherr A. (2010) Sozialarbeitswissenschaft, in: Grundriss Soziale Arbeit, W. Thole (ed.), 3. überarb. u. erw. Aufl., Wiesbaden, VS Verlag: 283-296.

Stichweh R. (1993) Wissenschaftliche Disziplinen: Bedingungen ihrer Stabilität im 19. und 20. Jahrhundert, in: Sozialer Raum und akademische Kulturen, in: J. Schriewer, E. Keiner, C. Charle (ed.), Frankfurt a.M., Lang: 235-250.

Stichweh R. (1994) Wissenschaft, Universität, Professionen. Soziologische Analysen, Frankfurt a.M., Suhrkamp.

Stisser A., Horn K.-P. (2012) Bachelor- und Masterstudiengänge in der Erziehungswissenschaft, in: Datenreport Erziehungswissenschaft 2012, in: W. Thole, H. FaulstichWieland, K.-P. Horn, H. Weishaupt, I. Züchner (ed.), Opladen, Barbara Budrich: 19-48.

Winkel R. (1989) Gespräche mit Pädagogen. Bildung - Erziehung - Schule, Weinheim, Beltz.

Vogel P. (1994) Klassifikationsprobleme als Ausdruck des defizitären Charakters der Erziehungswissenschaft?, in: Systematiken und Klassifikationen in der Erziehungswissenschaft, K.-P. Horn, L. Wigger (ed.), Weinheim, Deutscher Studien Verlag: 371-387.

Züchner I., Rauschenbach T. (2016) Erziehungswissenschaft - Beobachtungen zur Karriereeiner Disziplin, in: Kritische Bildungsforschung. Standortbestimmungen und Gegenstandsfelder, A. Schippling, C. Grunert, N. Pfaff (ed.), Opladen, Barbara Budrich: 124-146. 
Cathleen Grunert, Katja Ludwig

\section{Nauka o wychowaniu w Niemczech: zmiany w trybie studiowania i statusie dyscypliny w efekcie reform bolońskich}

Artykuł poświęcony jest europejskiej reformie szkolnictwa wyższego, koncentruje się na tzw. procesie bolońskim, w efekcie którego dokonano na niemieckich uniwersytetach głębokiej przemiany wcześniejszych studiów dyplomowych i magisterskich na studia licencjackie (Bachelorstudiengänge) i (nowe) magisterskie. Autorki skupiają się na zmianach mapy studiów z zakresu uniwersyteckiej nauki o wychowaniu (Erziehungswissenschaft) w Niemczech w trakcie reform bolońskich. Stawiają pytanie o konsekwencje wprowadzenia systemu studiów dwustopniowych dla instytucjonalnej organizacji kształcenia na kierunkach zogniskowanych na nauce o wychowaniu (Erziehungswissenschaft), a tym samym dla jej pozycji w niemieckim systemie szkolnictwa wyższego.

Przedmiotem badań, których wyniki są prezentowane w niniejszym artykule, były programy studiów i sylabusy wszystkich (głównych) kierunków studiów z zakresu nauki o wychowaniu, oferowanych w Niemczech w semestrze zimowym roku akademickiego 2014/2015. Poddano je ilościowej analizie treści. Poza cechami strukturalnymi kierunków (studia jedno- czy dwukierunkowe, licencjat, magisterium) i nazwami konkretnych przedmiotów, zwracano także uwagę na treści programowe (np. udział treści odnoszących się do dyscypliny, do metodologii badań).

Wyniki analizy ilościowej jednoznacznie pokazały, że polityczne procesy reorganizacji szkolnictwa wyższego wynikające z reform bolońskich znacząco zmieniły instytucjonalną organizację nauki o wychowaniu. Po pierwsze, obserwuje się dużą heterogeniczność modeli studiów w różnych ośrodkach, która ujawnia się już na poziomie cech strukturalnych. Po drugie, procesy różnicowania są widoczne w nazwach przedmiotów w ramach kierunków, a nazwy należy traktować jako symboliczne oznaczenie granic. Procesy te wskazują na wzrastającą specjalizację w subdyscyplinach już na poziomie studiów licencjackich, a jednocześnie zwracają uwagę na rozproszenie dyscyplinarnego etykietowania (Labeling), czemu towarzyszą problemy dyscyplinarnego przyporządkowania. W rezultacie czego w niektórych nazwach kierunków studiów (odwołujących się w większym lub mniejszym stopniu do pedagogiki) nie występuje ani pedagogika, ani nauki o wychowaniu. Po trzecie zaznacza się wzrost programowego (treściowego) zróżnicowania kierunków studiów, tzn. udziały treści z zakresu teoretycznych podstaw nauk o wychowaniu oraz metod badawczych są tak dalece odmienne w programach nowych kierunków studiów, że trudno wskazać odniesienie do wspól- 
nego dyscyplinarnego sedna. W tym kontekście program obejmujący treściowe minimum dla tworzonych kierunków studiów, wypracowany przez Deutschen Gesellschaft für Erziehungswissenschaft w celu utrzymania porównywalności oraz przyporządkowania kierunków studiów do dyscypliny nauk o wychowaniu, wydaje się niewiele znaczącym punktem odniesienia dla implementacji kierunków w niemieckich szkołach wyższych.

W końcowej części artykułu autorki podejmują dyskusję, w jakiej mierze wyraźne rozproszenie i zróżnicowanie kierunków może oddziaływać na pozycję dyscypliny w ramach systemu szkolnictwa wyższego oraz na jej wewnętrzny rozwój, ponieważ kierunki studiów należy traktować także jako pierwszy stopień kształcenia naukowego, a tym samym jako początek kształcenia młodej kadry.

Tłumaczenie: Danuta Urbaniak-Zając 